\title{
Stromal fibroblasts in the microenvironment of gastric carcinomas promote tumor metastasis via upregulating TAGLN expression
}

\author{
Beiqin Yu ${ }^{1}$, Xuehua Chen ${ }^{1}$, Jianfang $\mathrm{Li}^{1}$, Ying Qu${ }^{1}$, Liping Su${ }^{1}$, Yibing Peng ${ }^{2}$, Jian Huang ${ }^{3}$, Jun Yan ${ }^{4}$, Yingyan Yu ${ }^{1}$,
} Qinlong $\mathrm{Gu}^{1}$, Zhenggang Zhu' and Bingya Liu'

\begin{abstract}
Background: Fibroblasts play a critical role in tumorigenesis, tumor progression and metastasis. However, their detailed molecular characteristics and clinical significance are still elusive. TAGLN is an actin-binding protein that plays an important role in tumorigenesis.

Results: We investigated the interaction between cancer cells and the tumor microenvironment to determine how the fibroblasts from human gastric carcinoma facilitate tumorigenesis through TAGLN. QRT-PCR and Western blot indicated that TAGLN expression was upregulated in gastric carcinoma-associated fibroblasts (CAFs) that promote gastric cancer cell migration and invasion. Using small interfering RNA (siRNA), we found that CAFs enhanced tumor metastasis through upregulated TAGLN in vitro and in vivo. The expression of matrix metalloproteinase-2 (MMP-2) was significantly lower after TAGLN knock-down by siRNA. TAGLN levels were elevated in human gastric cancer stroma than normal gastric stroma and associated with differentiation and lymph node metastasis of gastric cancer.
\end{abstract}

Conclusion: CAFs may promote gastric cancer cell migration and invasion via upregulating TAGLN and TAGLN induced MMP-2 production.

Keywords: TAGLN, Fibroblast, Microenvironment, Tumor metastasis, Gastric carcinoma

\section{Background}

Gastric carcinoma is the second most common causes of cancer-related deaths in the world, especially in Asia [1,2]. Most of gastric cancer patients died of tumor recurrence caused by distant metastasis. Metastasis is a multi-stage process by which cancer cells disseminate from the primary neoplasm and invade surrounding tissue and distance organs, involving cancer cell motility, intravasation, transit in the blood or lymph and extravasation [3]. This process depends tightly on the surrounding microenvironment and tumor development relies on a continuous cross-talk between cancer cells and extracellular microenvironments [4].

\footnotetext{
* Correspondence: byliu@sjtu.edu.cn

'Key Laboratory of Shanghai Gastric Neoplasms, Department of Surgery, Shanghai Institute of Digestive Surgery, Ruijin Hospital, Shanghai Jiao Tong University School of Medicine, Shanghai 200025, China

Full list of author information is available at the end of the article
}

Transgelin (TAGLN, also known as SM22) is an actin cross-linking protein that is involved in calcium interactions and regulates contractile properties [5]. It may play a role in cell differentiation, cell migration cell invasion and matrix remodeling by stabilizing the cytoskeleton through actin binding [6,7]. Overexpression of TAGLN protein has been observed in carcinomas of the stomach, liver, and esophagus, while decreased levels of TAGLN mRNA have been observed in breast and colon cancer cell lines and primary tumors [8].

It is generally accepted that tumor-stroma interactions play an important role in tumor development and progression. The stroma is constituted mainly of extracellular matrix (ECM) and cellular elements, such as fibroblasts $[9,10]$. The cellular components of the stroma are well-recognized as having a supportive role in carcinogenesis $[11,12]$. These stromal elements act in a synergistic cross-talk with cancer cells from the primary sites to 

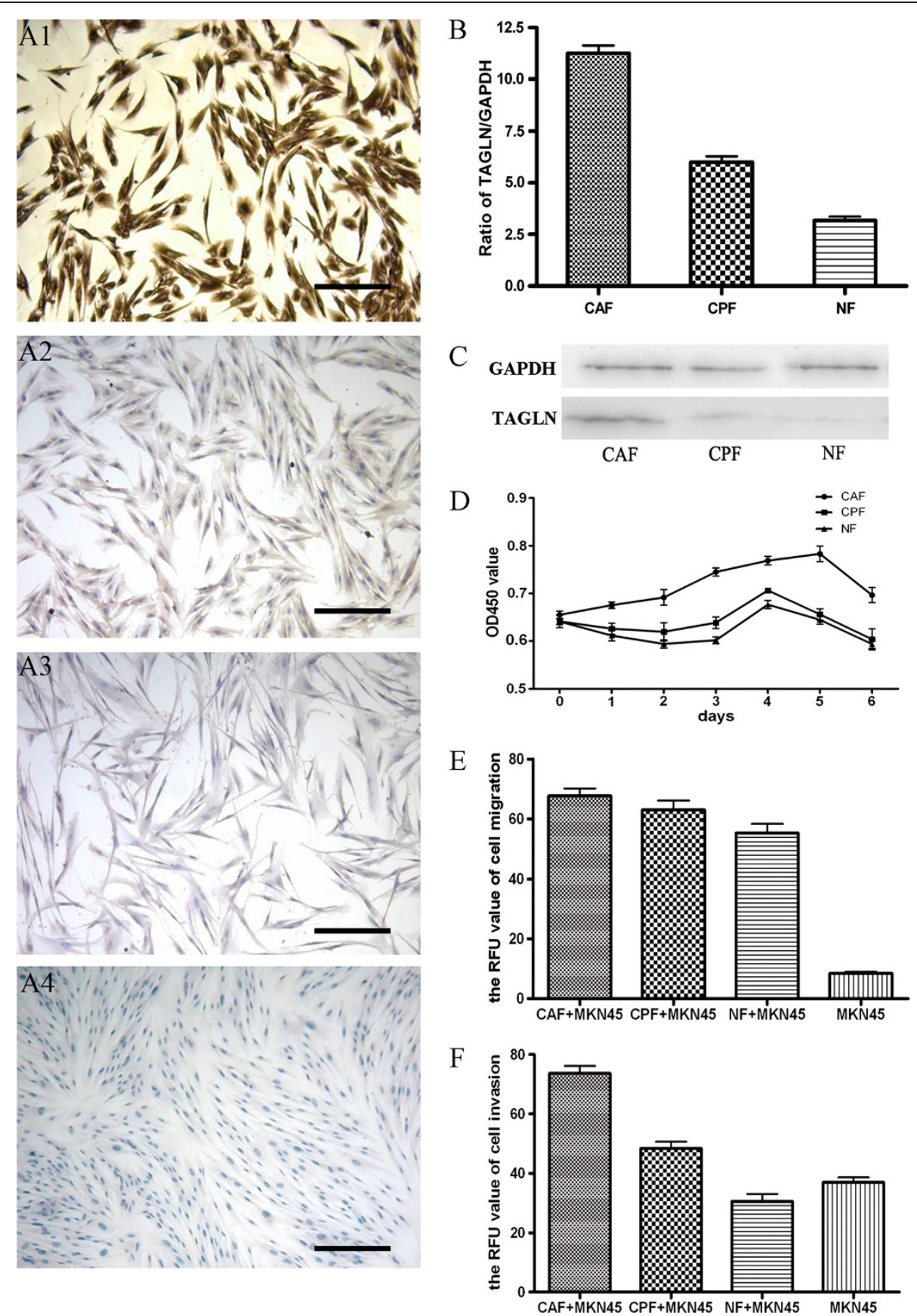

Figure 1 The expression of TAGLN is upregulated in gastric cancer-associated fibroblasts. A: characterization of gastric fibroblasts (ICC). a1:

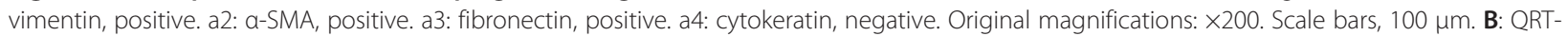
PCR analysis of TAGLN in CAF, CPF and NF. ${ }^{*}, P<0.05,{ }^{* *}, P<0.01$, compared to NF. C: Western blot analysis of TAGLN in CAF, CPF and NF, TAGLN expression level was decreased successively. $\mathbf{D}$ : fibroblasts growth curve were determined by CCK-8 assay, CAFs grew quickier than the others. E: CAF had higher ability to enhance MKN-45's migration ability than CPF and NF, ${ }^{* * *}, P<0.001$ compared to MKN-45. F: CAF had higher ability to enhance MKN-45's invasion ability than CPF and NF, ${ }^{*}, P<0.05,{ }^{* *}, P<0.01$, compared to MKN-45.

sustain cancer growth and metastasis [13,14]. The tumor stroma which is referred to as a "reactive stroma" is associated with an increased number of fibroblasts, enhanced capillary density and deposition of a new ECM rich in type-1-collagen and fibrin [15]. Accumulating evidence reveals that stromal cells, such as fibroblasts have a more profound influence on the development and progression of carcinoma than was previously appreciated [15-17].

Fibroblasts are well-known to play roles in tumorigenesis and progression. Fibroblasts express vimentin, fibronectin, 
$\alpha$-smooth-muscle actin ( $\alpha$-SMA), fibroblast surface protein as well as fibroblastic markers. Carcinomaassociated fibroblasts (CAFs) are activated fibroblasts, biologically different from fibroblasts present in benign microenvironments in several important aspects $[17,18]$. CAFs are not passive bystanders in tumorigenesis and metastasis, but contribute actively to these processes [19]. Our knowledge on the role of resting and activated fibroblasts in cancer is still evolving. Here, we focus on the interaction between cancer cells and their microenvironments to study how the fibroblasts isolated from human gastric carcinomas facilitate tumorigenesis.

\section{Results}

The expression of TAGLN is upregulated in gastric carcinoma-associated fibroblasts

The carcinoma-associated fibroblasts (CAFs), counterpart fibroblasts (CPFs) and normal fibroblasts (NFs) were obtained from primary tissue culture using tissues from cancer-associated regions, non-cancer-associated stroma and normal mucosa, respectively. We then verified the purity of the various fibroblast populations by immunostaining. These fibroblast populations expressed high levls of fibroblastic markers such as vimentin, $\alpha$-SMA and fibronectin. On the other hand, these cells did not express epithelial markers such as cytokeratin (Figure 1A). These data confirmed the purity of the fiborblasts. These observations indicate that the culture cells were predominantly fibroblasts were prepared with minimal contamination by other cells.

The mRNA and protein levels of TAGLN were lower in CPFs and NFs compared with CAFs (Figure $1 \mathrm{~B}$ and C). We first showed that CAFs grew significantly more than CPFs and NFs in vitro cell proliferation (Figure 1D). Next, we carried out cell invasion and migration assay to test whether the invasion and migration ability of gastric cancer cells would be enhanced by fibroblasts. The experiment was designed to investigate the effects of stroma TAGLN, which was secreted by fibroblasts, on human gastric cancer cells. The internal levels of TAGLN in gastric cancer cells might interfere the experimental process. Hence, we choosed MKN-45 cell line, because its TAGLN expression level was the lowest in seven gastric cancer cell lines (SNU-1, AGS, NCI-N87, KATOIII, MKN-45, MKN-28 and SGC-7901; data not shown). Interestingly, the invasion and migration ability of MKN-45 increased in all the three groups (CAFs, CPFs and NFs), and increased most in the group with CAF

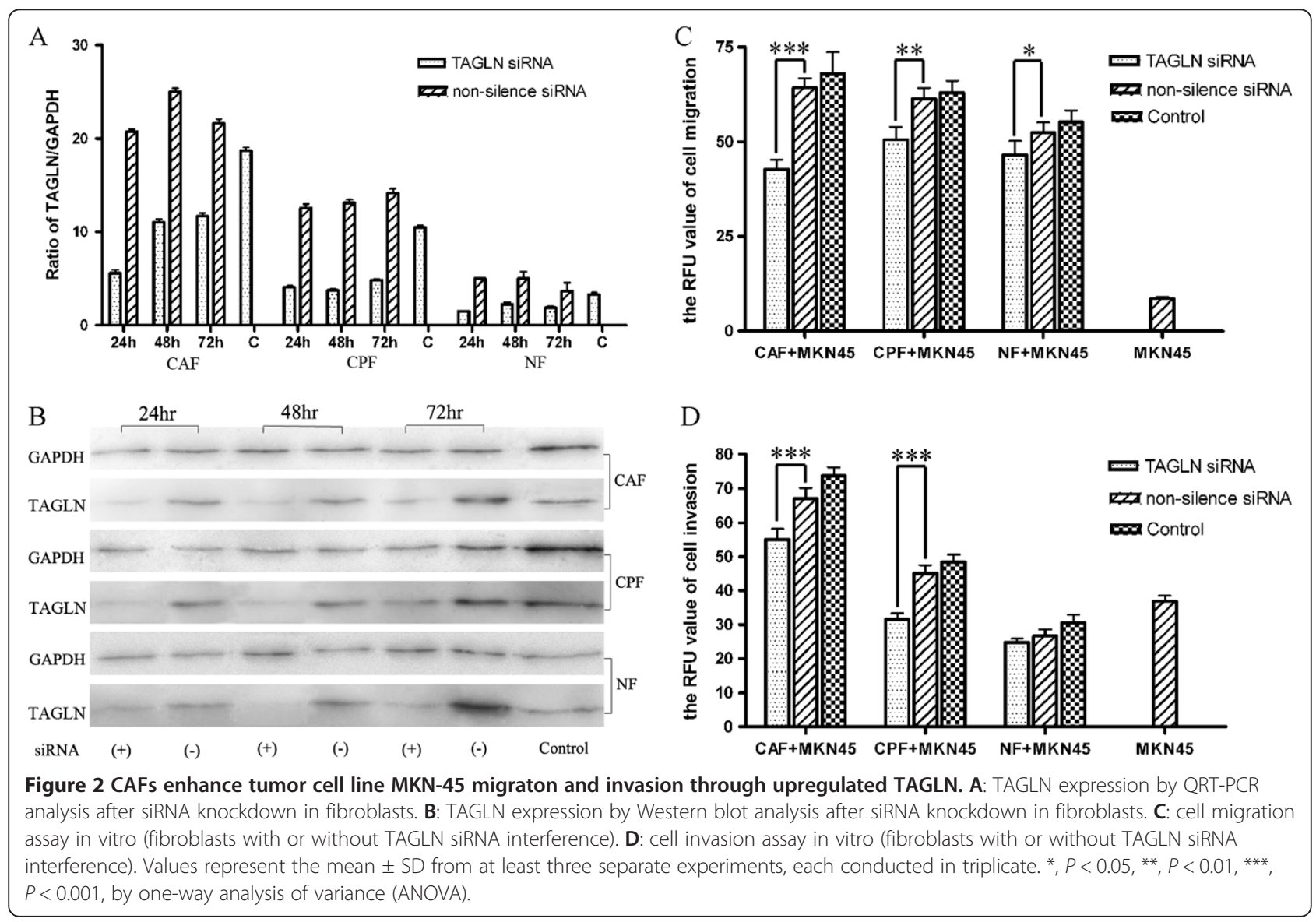


Table 1 Nude mice lung metastatic nodules in various groups through tail intravenous injection (Each group contained 6 mice)

\begin{tabular}{lcccccc}
\hline groups & $\begin{array}{c}\text { TAGLN siRNA } \\
\text { CAF-MKN45 }\end{array}$ & $\begin{array}{c}\text { non-silence siRNA } \\
\text { CAF-MKN45 }\end{array}$ & CAF-MKN45 & CPF-MKN45 & NF-MKN45 & MKN45 alone \\
\hline Number of tumor-bearing mice & 2 & 5 & 10 & 5 & 3 & 2 \\
Number of metastatic nodules & 2 & 9 & 5 & 3 & 2 \\
\hline
\end{tabular}

(Figure $1 \mathrm{E}$ and $\mathrm{F}$ ). The acquisition of migration and invasive behavior is one of steps in the metastatic process.

\section{CAFs promote tumor metastasis through upregulating TAGLN}

To examine the effect of TAGLN in fibrobalsts, we used RNAi to knock down TAGLN expression. CAFs, CPFs and NFs were transfected with TAGLN-specific or non-silence siRNA. RNA and protein were obtained at 24, 48 or $72 \mathrm{hrs}$ after transfection, TAGLN mRNA and protein levels were investigated by QRT-PCR and Western blot. TAGLN mRNA and protein were significantly inhibited in cells treated with TAGLN-specific siRNA (Figure 2A and B).

We next investigated whether the invasion and migration ability of gastric cancer cells will be enhanced by the expression of TAGLN in fibroblasts, we carried out cell invasion and migration assay in vitro. The fibroblasts and MKN-45 were non-contactedly co-cultured. Interestingly, the invasion and migration ability of MKN-45 decreased immediately in the group with CAFs and decreased slightly in the group with CPFs and NFs. The results showed that the invasion and migration ability of MKN45 can decrease through down-regulating the expression of TAGLN (Figure 2C and D).

In order to assess the contribution of CAFs-TAGLN to tumor metastasis in vivo, we emplyed a xenograft model.
We mixed TAGLN siRNA CAFs, non-silenced CAFs, CPFs and NFs with MKN-45 human gastric cancer cells in a 2:1 ratio or MKN-45 alone and inoculated these cells through tail intravenous injection in immunodeficient nude mice. After 6 weeks, MKN-45 mixed with CAFs generated more lung metastatic nodules than MKN-45 mixed with TAGLN siRNA CAFs, CPFs and NFs, similar to MKN-45 mixed with non-silence siRNA CAFs (Table 1). These data suggested that CAFs show an increased ability to stimulate tumor metastasis (Figure 3A and B). All the observations indicated that in the presence of CAFs, tumors became more aggressive, suggesting that CAFs might play roles in promoting metastasis in gastric cancer.

TAGLN promotes tumor metastasis by upregulating MMP-2 Many studies have focused on the role of matrix metalloproteinases (MMPs) to the invasion of surrounding connective tissue and metastasis of tumor cells from the primary lesion to distant sites in tumor progression $[20,21]$. In our previous work, we examined MMP-1, MMP-2, MMP-3, MMP-7 and MMP-9 levels after TAGLN knock-down by siRNA through ELISA, only MMP-2 level was significantly down-regulated, other MMPs levels showed no change. Hence, we focued on MMP-2 in this study. As Figure 4A showed, compared to MKN-45 co-coultured with CAF/neg and CAF/mock, MMP-2 levels in supernatant from MKN-45 co-cultured with
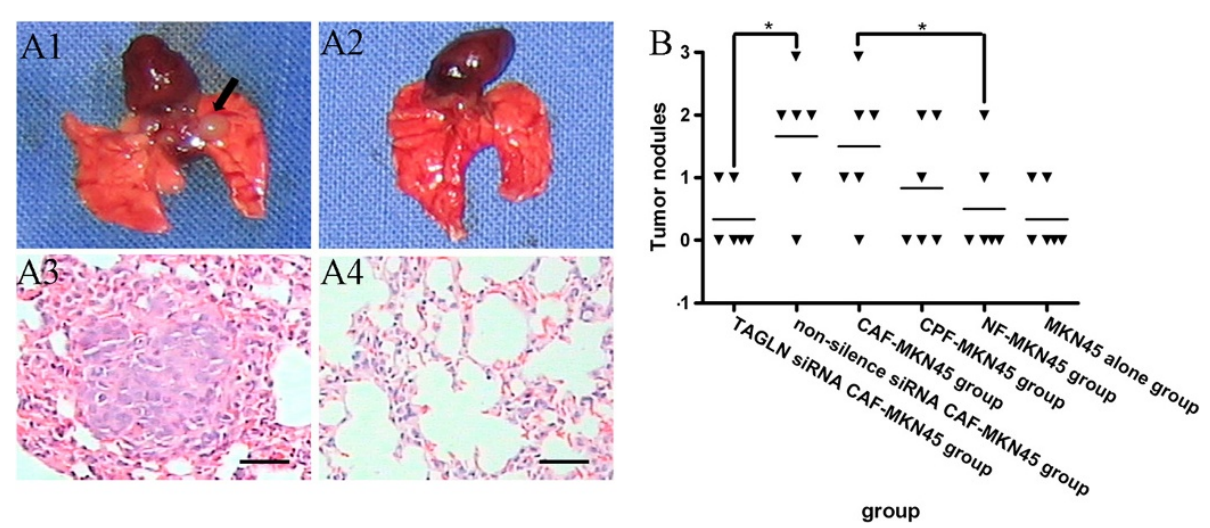

Figure 3 TAGLN enhances tumor metastasis through human tumor xenograft model in vivo. A: nude mice metastatic nodules in the lung. a1: lung metastatic nodules from tomur-bearing mouse. Arrow: metastatic nodule. a2: no metastatic nodule in lung. a3: H\&E staining for lung metastatic nodule under bright-field microscope. Scale bars, $200 \mu \mathrm{m}$. a4: H\&E staining for lung without metastatic nodule under bright-field microscope. Scale bars, $200 \mu \mathrm{m}$. B: quantification of nude mice lung metastatic nodules in various groups through tail intravenous injection. * $P<0.05$, by one-way analysis of variance (ANOVA). 
A

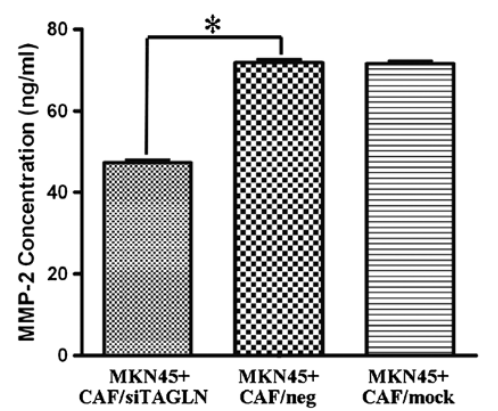

B

$72 \mathrm{kDa}$

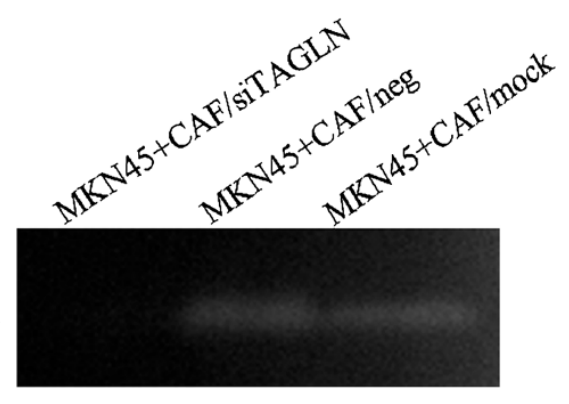

Figure 4 TAGLN enhances tumor metastasis by upregulated MMP-2. A: MMP-2 expression in CAF decresed obviously after TAGLN RNAi by ELISA. *, $P<0.05$ by one-way analysis of variance (ANOVA). Error bars depict the standard error around the mean. B: knockdown TAGLN impaired the ability of CAF to secret MMP-2 by gelatin zymography.

CAF/siTAGLN were significantly suppressed. We next examined the activity of MMP-2 using gelatin zymography assay. We found MMP-2 activity was dramatically inhibited in supernatant from MKN-45 co-cultured with CAF/siTAGLN than with $\mathrm{CAF} /$ neg and $\mathrm{CAF} / \mathrm{mock}$ (Figure 4B). Thus, TAGLN may enhance tumor metastasis through the MMP-2 enzymes degrade the basement membranes (eg. collagen IV).

TAGLN expression in human gastric cancer and its association with differentiation in gastric cancer patients To evaluate the TAGLN expression in gastric cancer, TAGLN immunostaining in 98 primary gastric cancer tissues were examined. TAGLN expression was upregulated in human gastric cancer stroma compared to normal gastric tissue (Figure 5A: normal gastric tissue; Figure 5B: gastric cancer tissue). The correlation of TAGLN expression with the clinicopathological features was shown in Table 2. TAGLN levels were higher in undifferentiated tumors (poorly differentiated adenocarcinomas, signet ring cell carcinomas and mucinous carcinomas) than that in differentiated ones (well and moderate adnocarcinomas) $(P=0.003)$. Moreover, TAGLN levels was correlated with lymph node metastasis $(P=0.029)$. There were no significant differences in tumor TAGLN expression according to the other clinicopathological features such as gender,

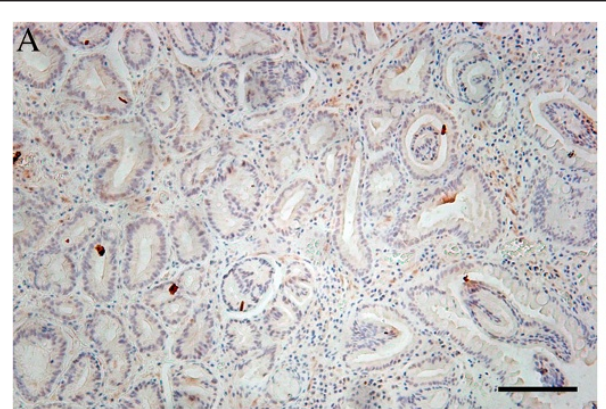

$\mathrm{C}$

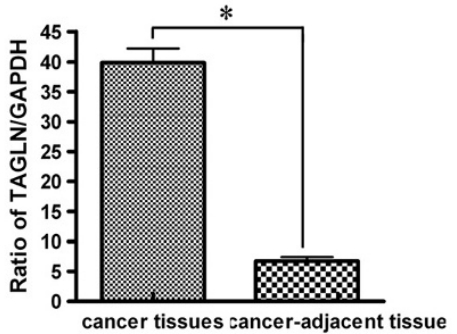

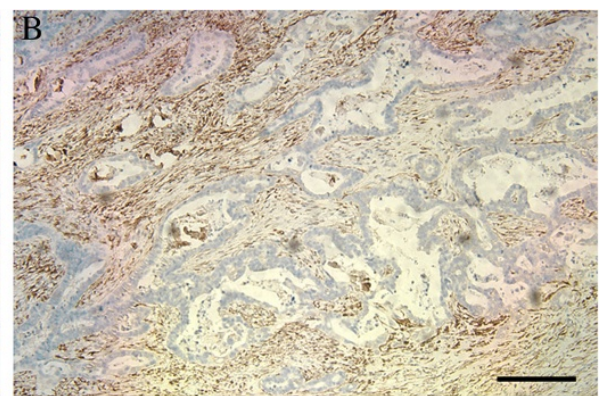

D

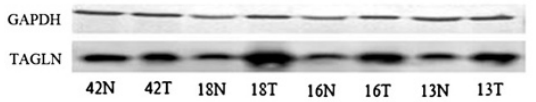

Figure 5 Expression of TAGLN in human gastric cancer tissues. A: TAGLN expression level in normal gastric tissue by IHC. Scale bars, $100 \mu$ m. B: TAGLN is overexpressed in the stroma of human gastric cancer by IHC. Scale bars, $100 \mu \mathrm{m}$. C: TAGLN mRNA is upregulated in human gastric cancer analyzed by QRT-PCR. D: TAGLN protein is upregulated in human gastric cancer analyzed by Western blot. Values represent the mean \pm SD from at least three separate experiments, each conducted in triplicate. ${ }^{*}, P<0.001$ by paried-samples $T$ test. 
Table 2 Clinicopathological associations of TAGLN expression in gastric cancer

\begin{tabular}{|c|c|c|c|c|}
\hline & Number & TAGLN in & nunostaining & $\mathbf{P}$ \\
\hline & & Weak(+) & Strong(++) & \\
\hline Gender & & & & 0.778 \\
\hline Male & 60 & 27 & 33 & \\
\hline Female & 38 & 16 & 22 & \\
\hline Age & & & & 0.739 \\
\hline$\leq 60 y$ & 46 & 21 & 25 & \\
\hline$>60 y$ & 52 & 22 & 30 & \\
\hline Tumor size & & & & 0.852 \\
\hline$\leq 5 \mathrm{~cm}$ & 58 & 25 & 33 & \\
\hline$>5 \mathrm{~cm}$ & 40 & 18 & 22 & \\
\hline Differentiation & & & & 0.003 \\
\hline Well + Moderate & 46 & 13 & 33 & \\
\hline Poor & 52 & 30 & 22 & \\
\hline Lauren classification & & & & 0.059 \\
\hline Intestinal & 40 & 13 & 27 & \\
\hline Diffuse & 58 & 30 & 28 & \\
\hline T stage & & & & 0.142 \\
\hline $\mathrm{T} 1+\mathrm{T} 2$ & 56 & 21 & 35 & \\
\hline $\mathrm{T} 3+\mathrm{T} 4$ & 42 & 22 & 20 & \\
\hline Lymph node metastasis & & & & 0.029 \\
\hline No & 27 & 11 & 16 & \\
\hline N1 & 32 & 15 & 17 & \\
\hline $\mathrm{N} 2$ & 26 & 7 & 19 & \\
\hline N3 & 13 & 10 & 3 & \\
\hline Distant metastasis & & & & 0.709 \\
\hline Without & 95 & 42 & 53 & \\
\hline With & 3 & 1 & 2 & \\
\hline TNM stage & & & & 0.722 \\
\hline I & 20 & 7 & 13 & \\
\hline$\|$ & 25 & 11 & 14 & \\
\hline III & 30 & 10 & 20 & \\
\hline IV & 23 & 15 & 8 & \\
\hline
\end{tabular}

age, tumor size, Lauren classification, $\mathrm{T}$ stage, distance metastasis and TNM stage $(P \geq 0.05)$.

Also, we used QRT-PCR and Western blot to evaluate the expression of TAGLN in gastric cancer tissues (Figure 5C and D). These results suggested that the TAGLN expression in gastric cancer tissues was much higher in gastric cancer than that in paired normal tissues.

\section{Discussion}

Accumulating evidence demonstrates that cancer initiation and progression involve interactions between both tumor and stromal cells. Tumor cells can actively recruit stromal cells, such as vascular cells, smooth muscle cells and fibroblasts, into the tumor, thus generating microenvironment to foster tumor growth $[16,22,23]$. Fibroblasts often constitute the majority of the stromal cells within a gastric carcinoma, yet the functional contributions of these cells to tumorigenesis and tumor metastasis remain poorly understood.

TAGLN is one of the earliest markers of smooth muscle differentiation during embryogenesis [24], and previous studies related to TAGLN mainly focused on smooth muscle cells [25]. Pathological conditions accompanied with tissue remodeling and fibrogenesis, such as wound healing and neoplastic lesions, are characterized by the appearance of stromal cells with ultrastructural features intermediate between those of typical fibroblasts and those of smooth muscle cells. These fibroblasts exhibit phenotypic and functional features of smooth muscle cells and hence are named myofibroblasts [26], such as CAFs. In Our study, TAGLN overexpression was also observed in CAFs. It is reported that the TAGLN protein is overexpressed in gastric cancer when protein expression spectrum of gastric cancer was analyzed using twodimensional gel electrophoresis (2-DE) [27]. Furthermore, the TAGLN protein is one of the two tumor-associated antigens which were identified in serum of kidney carcinoma patients by Klade using 2-DE [28]. These reports are consistent with the findings that we have observed using real-time PCR and Western Blot. Interestingly, we found that TAGLN was mainly detected in fibroblasts derived from tumor stroma, rather than in tumor cells. Similarly, other investigators discovered that the TAGLN is not expressed in the malignant cells but in mesenchymal cells of the tumor stroma [27].

Stromal fibroblasts regulate endothelial or epithelial cell behavior through direct and indirect cell-cel1 interactions. Activation of host stroma1 microenvironment is thought to be a critical step in tumor growth and progression $[13,29]$. Proteins secreted by stromal cell in the tumors may positively or negatively affect tumor progression. The most prominent actions of TAGLN may favor tumor cell motility and invasion [7,30,31]. We found that the TAGLN was significantly increased in lymph node metastasis group than lymph node negative group. To c1arify the role of TAGLN in stromal fibroblasts during gastric cancer tumorigenesis and metastasis, we silenced the TAGLN expression in human CAFs. It was indicated that only the MKN-45 co-cultured with CAFs expressing high level TAGLN exhibited much higher invasion and migration ability. Fibroblasts which TAGLN had been knocked down did not display this function. Taken together, we suggest that TAGLN might be responsible for the enhancement of gastric cancer cell metastasis via stromal cells such as CAFs. 
TAGLN expression is increased significantly in gastric CAFs. CAFs with increased TAGLN expression levels may enhance tumor cell invasion and migration ability which promote an enhanced expression of MMP-2. The stimulation of MMP expression in fibroblasts either after direct contact with tumor cells or after contact with tumor cell-derived soluble factors from tumors originating from epithelial cells has been shown in several studies [32-35]. Clarifying the underlying mechanisms may therefore attenuate the tumor recurrence and metastasis in gastric cancer patients.

\section{Conclusions}

In summary, our study demonstrates that CAFs may promote gastric cancer cell migration and invasion via increased MMP-2 production caused by TAGLN upregulation.

\section{Methods}

\section{Tissue samples}

A total of 40 pairs of cancer tissues and corresponding normal mucosa for QRT-PCR and Western blot and 98 primary gastric cancers for immunostaining were collected from surgically removed gastric cancer diagnosed at the Department of Surgery, Ruijin hospital, Shanghai Jiao Tong University School of Medicine. Normal mucosa samples were taken from the margin of the resection where the tumors located at least over $6 \mathrm{~cm}$ apart from it. This study protocol was approved by the independent ethics committee of Ruijin Hospital, Shanghai Jiaotong University, School of Medicine. The informed consent was written. All samples were snap frozen in liquid nitrogen overnight and kept at $-80^{\circ} \mathrm{C}$ before use. $4 \%$ Formaldehyde-fixed tissue sections of both tumor and mucosa were stained with $\mathrm{H} \& \mathrm{E}$ and examined and verified histopathologically by two pathologists. Each specimen was attributed a diagnosis and scored for Lauren classification, differentiation, pTNM stage. Tumor size, location and other clinical pathological characteristics were obtained from clinical records with patient permission.

\section{Cell lines}

Gastric cancer cell lines SNU-1 (ATCC: CRL-5971), AGS (ATCC: CRL-1739), NCI-N87 (ATCC: CRL-5822) and KATOIII (ATCC: HTB-103) were obtained from the American Type Culture Collection (Manassas, VA, USA). Another 3 gastric cancer cell lines, MKN-45, MKN-28 and SGC-7901, were preserved at our institute. All cell lines were maintained in RPMI-1640 supplemented with $10 \%$ fetal bovine serum (FBS).

\section{Antibodies}

Primary antibodies used for staining included human specific anti-vimentin (Abcam, Cambridge, UK), anti- fibronectin (Abcam, Cambridge, UK), anti- $\alpha$-smoothmuscle actin (Sigma Chemical, St Louis, MO, USA), antipan-cytokeratin (Abcam, Cambridge, UK), anti-SM22 (Abcam, Cambridge, UK), and mouse anti-GAPDH (Kangchen, China).

\section{Isolation of human gastric fibroblasts}

Fibroblasts were isolated from cancer- and non-cancer-associated regions of gastric tissues dissected from patients with gastric cancer during radical gastric resection from the Department of Surgery, Ruijin hospital, Shanghai Jiao Tong University School of Medcine. The cancer-associated regions were selected to be minimally necrotic regions of the tumor mass. Non-cancer-associated stroma, which was isolated from tissue at least $2 \mathrm{~cm}$ distal to the outer margin of the cancer mass. Normal mucosa samples were taken from the margin of the resection where the tumors located at least over $6 \mathrm{~cm}$ apart from it. Tissues were minced into organoids of approximately $1 \mathrm{~mm}^{3}$ and seeded on uncoated plastic material in RPMI 1640 medium containing human basic fibroblast growth factor, $20 \%$ fetal calf serum (FCS) and penicillin and streptomycin as antibiotics. These conditions produced a homogenous fibroblastic cell population after 7 days of culture. Each fibroblast was then expanded at a 1:3 ratio by trypsin-EDTA into $10 \mathrm{~cm}$ petri dishes. We used fibroblasts passaged for up to 8 population doublings (PDs) for subsequent experiments, in order to minimize clonal selection and culture stress which could occur during extended tissue culture.

\section{Immunohistochemical staining (IHC)}

Cells or tissues were fixed for 30 min with $4 \%$ formalin and rinsed with phosphate buffered saline (PBS). After endogenous peroxidase activity was quenched with $0.3 \%$ $\mathrm{H}_{2} \mathrm{O}_{2}$, cells were blocked with normal nonimmune serum for $30 \mathrm{~min}$ prior to being incubated with primary antibody at $37^{\circ} \mathrm{C}$ for $2 \mathrm{hrs}$. Cells or tissues were then labeled with streptavidin-biotin-horseradish peroxidase staining kit (Dako, Carpinteria, CA, USA). The presence of labeled peroxidase on the sections was visualized by incubation with $3,3^{\prime}$-diaminobenzidine, DAB + substrate chromogen and the sections were counterstained with hematoxylin. Negative control slides were performed by omission of the primary antibody.

TAGLN expression was assessed by the intensity of stained cells, and determined in two categories (weak positive and strong positive). The staining intensity was classified according to four grades (intensity scores): no staining (grade 0), light brown staining (grade 1), brown staining (grade 2), and dark brown staining (grade 3). Grade 0 and grade 1 was defined as weak positive, grade 2 and grade 3 were defined as strong positive. 


\section{Quantitative real-time PCR (QRT-PCR)}

Total RNA was extracted using Trizol reagent (Invitrogen, Carlsbad, CA, USA) following the manufacturer's instructions. cDNA was obtained with $1 \mu \mathrm{g}$ RNA using Reverse Transcription System Kit (Promega, Madison, WI, USA) and QRT-PCR was performed by the SYBR Green PCR core Reagent kit (Applied Biosystems, Warrington, UK). Primers specific to TAGLN were as follows: $5^{\prime}$-GAGCA AGCTGGTGAACAGCC-3' (upper), 5'-GACCATGGAG GGTGGGT TCT-3' (lower). Glyceral-dehyde-3-phosphate dehydrogenase (GAPDH) was used as the endogenous reference. Its sequences were 5'-GGACCTGACCTGCC GTCT AG-3' (upper), 5'-GTAGCCCAGGATGCCCTT GA-3' (lower). QRT-PCR for quantitation of the mRNA levels of TAGLN was done on an ABI Prism 7000 (Applied Biosystems, Foster City, CA, USA). Data were analyzed by using the comparative $\mathrm{Ct}$ method. Specificity of resulting PCR products was confirmed by melting curves.

\section{Western blot}

Cells were harvested and lysed with mammalian protein extraction reagent (Pierce, Rockford, IL, USA). Protein concentrations were determined with a bicinchoninic acid (BCA) protein assay kit (Pierce, Rockford, IL, USA). Samples containing $50 \mu \mathrm{g}$ of total protein were loaded onto each lane of $12 \%$ acrylamide gel in a minigel apparatus (Bio-Rad, Richmond, CA, USA). The separated proteins were transferred to a polyvinylidene difluoride (PVDF) membrane (Bio-Rad, Richmond, CA, USA). After being incubated with primary antibody $(1: 1,000)$ and HRP-conjugated secondary antibody $(1: 5,000)$ respectively, immune complexes were detected by the enhanced chemiluminescent (ECL) system (Millipore, Bedford, MA, USA).

\section{Enzyme-linked immunosorbent assay (ELISA)}

The protein levels of MMP-2 in supernatants were measured by an ELISA kit (R\&D Systems Inc., Minneapolis, $\mathrm{MN}$, USA) according to the manufacturer's instructions.

\section{Cell growth assay}

The different groups of cells $\left(1 \times 10^{3}\right)$ were seeded into 96-well plates in triplicate. The number of viable cells was determined daily using the Cell Counting Kit (CCK-8) by utilizing a highly water-soluble tetrazolium salt WST-8 [2-(2-methoxy-4- nitrophenyl)-3-(4-nitrophenyl)-5(2,4-disulfophenyl)-2H-tetrazolium, monosodium salt] (Dojindo, Japan). Briefly, $20 \mu \mathrm{l}$ of CCK-8 solution was added into plates, absorbance at $450 \mathrm{~nm}$ was measured after 4 hrs incubation.

\section{Assessment of tumor invasion and migration}

Tumor invasion and migration assay were performed with QCMTM24-well invasion kit (Millipore, Bedford, MA, USA) and QCMTM24-well migration kit (Millipore, Bedford, MA, USA) according to the protocol provided by the manufacturer, respectively. Add prepared MKN-45 cell suspension $\left(2 \times 10^{5}\right.$ cells/well $)$ to the upper chamber and add pre-suspended fibroblasts $\left(5 \times 10^{4}\right.$ cells/well in RPMI 1640 medium containing 20\% FBS) to the lower chamber. The TAGLN of some fibroblasts in lower chambers was inhibited using siRNA 24 hrs later. MKN-45 and fibroblasts were co-cultured non-contactedly for $48 \mathrm{hrs}$, then lysised the cells and determined RFU values with a fluorescence plate reader using 480/520 nm filter set.

\section{TAGLN siRNA}

The Qiagen software was used to design RNAi sequences targeting human TAGLN (Accession no. NM_003186), and the siRNA sequence with the highest putative efficacy (sense: 5'-AAAUCGAGAAGAAGUA UGAdtdt-3', antisense: 5'-UCAUAC UUCUUCUCGAU UUdtdt- $3^{\prime}$ ) was synthesized by Shanghai GeneChem Co, Ltd. siRNA with randomized sequence (sense: $5^{\prime}$-UUCU CCGAACGUGUCACG Utt-3', antisense: $5^{\prime}$-ACGUGAC ACGUUCGGAGAAtt-3') against no gene (scrambled siRNA group) was transfected as internal control. For experiments, cells were transfected with the DOTAP liposomal transfection reagent (Roche, Germany). Cells were seeded at $2 \times 10^{5}$ cells/well in 6-well plates. After $24 \mathrm{hrs}$ when cells were in the phase of log growth, $250 \mu \mathrm{l}$ Opti-MEM I was mixed with $5 \mu \mathrm{l}$ of $20 \mu \mathrm{M}$ siRNA duplex, while another $250 \mu \mathrm{l}$ Opti-MEM I was separately incubated with $11.88 \mu \mathrm{l}$ of DOTAP liposome. The two mixtures were gently mixed, and incubated for about $30 \mathrm{~min}$ at room temperature. For transfection, the entire mixture was added to each well in $1.5 \mathrm{ml}$ of fresh medium without antibiotics. The final transfected concentration of siRNA was $20 \mathrm{nM}$. Cells were collected for further assay at 24,48 and $72 \mathrm{hrs}$ after transfection.

\section{Gelatin zymography}

The culture supernatants were harvested at $24 \mathrm{hrs}$ and mixed with a gel sample buffer $(0.5 \mathrm{M}$ Tris- $\mathrm{HCl}$, glycerol, $10 \%$ sodium dodecyl sulfate [SDS], $\beta$-mercaptoethanol, and $0.5 \%$ bromophenol blue). Ten micrograms of protein were taken by SDS-PAGE separation; the SDS-PAGE gels contained $0.1 \%$ gelatin (Sigma Chemical, St Louis, MO, USA). After electrophoresis, the gels were washed in $50 \mathrm{mM}$ Tris buffer containing 2.5\% Triton X-100. The gels were incubated for an additional $24 \mathrm{hrs}$ in incubation fluid (50 mM Tris buffer [pH 7.6], $10 \mathrm{mM} \mathrm{CaCl}_{2}$, and $200 \mathrm{mM}$ $\mathrm{NaCl})$. The gels were stained with $0.5 \%$ Coomassie blue, white bands $(72 \mathrm{kDa})$ on a blue background indicated zones of digestion corresponding to the presence of MMP-2. 


\section{Mouse model}

Female BALB/c nu/nu nude mice, age-matched between 4-5 weeks (Institute of Zoology Chinese Academy of Sciences), were housed at a specific pathogen-free environment in the Animal Laboratory Unit, Shanghai Jiao Tong University School of Medicine, China. Fibroblasts and MKN-45 were mixed at the ratio of 1:4 within $0.1 \mathrm{ml}$ PBS and injected into the lateral tail vein. Six mice were included in each group in all experiments, and each experiment was performed twice. Animals were sacrificed and lung metastatic nodules were recorded 6 weeks after tumor cell implantation. Tumor specimens were collected, mixed in formalin, embedded in paraffin, and subjected to $H \& E$ staining. Mice were manipulated and cared according to the guidelines and protocols approved by the Medical Experimental Animal Care Commission of Shanghai Jiaotong University School of Medicine.

\section{Statistical analysis}

All experiments were repeated at least three times. Results were summarized as means \pm standard deviation (SD). The correlation between TAGLN expression and clinicopathological parameters was calculated with two-sided chi-square test. $P<0.05$ was selected as the statistically signignificant value. SPSS version 11.0 software was used for all analyses.

\section{Competing interests}

The authors declare no competing interest in this paper.

\section{Authors' contributions}

$\mathrm{BQY}$ and $\mathrm{XHC}$ conducted the experiments. BQY and BYL made the hypothesis, designed the experiments and wrote the manuscript. JFL, YQ and LPS provided assistance in some of the experiments. BQY and YBP collect the specimens. JH, JY, YYY, QLG and ZGZ supervised the study. All authors read and approved the final manuscript.

\section{Acknowledgments}

This work was supported by the grands from National Natural Science Foundation of China (No. 30872476), National High Technology Research and Development Program of China (863 Program, No. 2006AA02A301, No. 2007AA02Z179), Science and Technology Commission of Shanghai Municipality (No. 09DZ1950100, No. 09DZ2260200), Shanghai Key Discipline (S30204) and Key Projects in the National Science \& Technology Pillar Program (No. 2008BA152B03).

\section{Author details \\ 'Key Laboratory of Shanghai Gastric Neoplasms, Department of Surgery, Shanghai Institute of Digestive Surgery, Ruijin Hospital, Shanghai Jiao Tong University School of Medicine, Shanghai 200025, China. ${ }^{2}$ Department of Laboratory Medicine, Ruijin Hospital, Shanghai Jiaotong University School of Medicine, Shanghai 200025, China. ${ }^{3}$ National Human Genome Center at Shanghai, Shanghai 201203, China. ${ }^{4}$ James Graham Brown Cancer Center, University of Louisville, Louisville, KY 40202, USA.}

Received: 21 November 2012 Accepted: 15 March 2013 Published: 19 March 2013

\section{References}

1. Eccles SA, Welch DR: Metastasis: recent discoveries and novel treatment strategies. Lancet 2007, 369(9574):1742-1757.

2. Plummer M, Franceschi S, Munoz N: Epidemiology of gastric cancer. IARC Sci Publ 2004, 157:311-326.
3. Chambers AF, Groom AC, MacDonald IC: Dissemination and growth of cancer cells in metastatic sites. Nature reviews 2002, 2(8):563-572.

4. Gout S, Huot J: Role of cancer microenvironment in metastasis: focus on colon cancer. Cancer Microenviron 2008, 1(1):69-83.

5. Assinder SJ, Stanton JA, Prasad PD: Transgelin: an actin-binding protein and tumour suppressor. Int J Biochem Cell Biol 2009, 41(3):482-486.

6. Thompson O, Moghraby JS, Ayscough KR, Winder SJ: Depletion of the actin bundling protein SM22/transgelin increases actin dynamics and enhances the tumourigenic phenotypes of cells. BMC cell biology 2012, 13:1.

7. Lee EK, Han GY, Park HW, Song YJ, Kim CW: Transgelin promotes migration and invasion of cancer stem cells. Journal of proteome research 2010, 9(10):5108-5117.

8. Rho JH, Roehrl MH, Wang JY: Tissue proteomics reveals differential and compartment-specific expression of the homologs transgelin and transgelin-2 in lung adenocarcinoma and its stroma. Journal of proteome research 2009, 8(12):5610-5618.

9. Korkaya H, Liu S, Wicha MS: Breast cancer stem cells, cytokine networks, and the tumor microenvironment. J Clin Investig 2011, 121(10):3804-3809.

10. Lorusso G, Ruegg C: The tumor microenvironment and its contribution to tumor evolution toward metastasis. Histochemistry and cell biology 2008, 130(6):1091-1103.

11. Bhowmick NA, Moses HL: Tumor-stroma interactions. Curr Opin Genet Dev 2005, 15(1):97-101.

12. Kitadai Y: Cancer-stromal cell interaction and tumor angiogenesis in gastric cancer. Cancer Microenviron 2010, 3(1):109-116.

13. Mueller MM, Fusenig NE: Tumor-stroma interactions directing phenotype and progression of epithelial skin tumor cells. Differentiation; research in biological diversity 2002, 70(9-10):486-497.

14. Mitra S, Stemke-Hale K, Mills GB, Claerhout S: Interactions between tumor cells and microenvironment in breast cancer: a new opportunity for targeted therapy. Cancer science 2012, 103(3):400-407.

15. Kalluri R, Zeisberg M: Fibroblasts in cancer. Nature reviews 2006, 6(5):392-401.

16. Bhowmick NA, Neilson EG, Moses HL: Stromal fibroblasts in cancer initiation and progression. Nature 2004, 432(7015):332-337.

17. Orimo A, Gupta PB, Sgroi DC, Arenzana-Seisdedos F, Delaunay T, Naeem R, Carey VJ, Richardson AL, Weinberg RA: Stromal fibroblasts present in invasive human breast carcinomas promote tumor growth and angiogenesis through elevated SDF-1/CXCL12 secretion. Cell 2005, 121(3):335-348.

18. Witz IP: Tumor-microenvironment interactions: dangerous liaisons. Adv Cancer Res 2008, 100:203-229.

19. Kiaris H, Chatzistamou I, Kalofoutis C, Koutselini H, Piperi C, Kalofoutis A: Tumour-stroma interactions in carcinogenesis: basic aspects and perspectives. Mol Cell Biochem 2004, 261(1-2):117-122.

20. Scherer RL, Mclntyre JO, Matrisian LM: Imaging matrix metalloproteinases in cancer. Cancer Metastasis Rev 2008, 27(4):679-690.

21. Kessenbrock K, Plaks V, Werb Z: Matrix metalloproteinases: regulators of the tumor microenvironment. Cell 2010, 141(1):52-67.

22. Cunha GR, Hayward SW, Wang YZ, Ricke WA: Role of the stromal microenvironment in carcinogenesis of the prostate. Int J Cancer 2003, 107(1):1-10.

23. Semba S, Kodama Y, Ohnuma K, Mizuuchi E, Masuda R, Yashiro M, Hirakawa $\mathrm{K}$, Yokozaki H: Direct cancer-stromal interaction increases fibroblast proliferation and enhances invasive properties of scirrhous-type gastric carcinoma cells. Br J Cancer 2009, 101(8):1365-1373.

24. Li L, Miano JM, Cserjesi P, Olson EN: SM22 alpha, a marker of adult smooth muscle, is expressed in multiple myogenic lineages during embryogenesis. Circ Res 1996, 78(2):188-195.

25. Lawson D, Harrison M, Shapland C: Fibroblast transgelin and smooth muscle SM22alpha are the same protein, the expression of which is down-regulated in many cell lines. Cell motility and the cytoskeleton 1997, 38(3):250-257

26. Mukaratirwa S, Koninkx JF, Gruys E, Nederbragt H: Mutual paracrine effects of colorectal tumour cells and stromal cells: modulation of tumour and stromal cell differentiation and extracellular matrix component production in culture. Int J Exp Pathol 2005, 86(4):219-229.

27. Ryu JW, Kim HJ, Lee YS, Myong NH, Hwang CH, Lee GS, Yom HC: The proteomics approach to find biomarkers in gastric cancer. Journal of Korean medical science 2003, 18(4):505-509. 
28. Klade CS, Voss T, Krystek E, Ahorn H, Zatloukal K, Pummer K, Adolf GR: Identification of tumor antigens in renal cell carcinoma by serological proteome analysis. Proteomics 2001, 1(7):890-898.

29. Zipin A, Israeli-Amit M, Meshel T, Sagi-Assif O, Yron I, Lifshitz V, Bacharach E, Smorodinsky NI, Many A, Czernilofsky PA, et al: Tumor-microenvironment interactions: the fucose-generating FX enzyme controls adhesive properties of colorectal cancer cells. Cancer Res 2004, 64(18):6571-6578.

30. Daniel C, Ludke A, Wagner A, Todorov VT, Hohenstein B, Hugo C: Transgelin is a marker of repopulating mesangial cells after injury and promotes their proliferation and migration. Laboratory investigation; a journal of technical methods and pathology 2012, 92(6):812-826.

31. Dos Santos Hidalgo G, Meola J, Rosa ESJC, Paro de Paz CC, Ferriani RA: TAGLN expression is deregulated in endometriosis and may be involved in cell invasion, migration, and differentiation. Fertil Steril 2011, 96(3):700-703

32. Guo H, Zucker S, Gordon MK, Toole BP, Biswas C: Stimulation of matrix metalloproteinase production by recombinant extracellular matrix metalloproteinase inducer from transfected Chinese hamster ovary cells. J Biol Chem 1997, 272(1):24-27.

33. Ito A, Nakajima S, Sasaguri Y, Nagase H, Mori Y: Co-culture of human breast adenocarcinoma MCF-7 cells and human dermal fibroblasts enhances the production of matrix metalloproteinases 1, 2 and 3 in fibroblasts. Br J Cancer 1995, 71(5):1039-1045.

34. Klein G, Vellenga E, Fraaije MW, Kamps WA, de Bont ES: The possible role of matrix metalloproteinase (MMP)-2 and MMP-9 in cancer, e.g. acute leukemia. Critical reviews in oncology/hematology 2004, 50(2):87-100.

35. Luo J: Role of matrix metalloproteinase- 2 in ethanol-induced invasion by breast cancer cells. J Gastroenterol Hepatol 2006, 21(Suppl 3):S65-S68.

doi:10.1186/1471-2121-14-17

Cite this article as: Yu et al:: Stromal fibroblasts in the

microenvironment of gastric carcinomas promote tumor metastasis via upregulating TAGLN expression. BMC Cell Biology 2013 14:17.

\section{Submit your next manuscript to BioMed Central and take full advantage of:}

- Convenient online submission

- Thorough peer review

- No space constraints or color figure charges

- Immediate publication on acceptance

- Inclusion in PubMed, CAS, Scopus and Google Scholar

- Research which is freely available for redistribution 